\title{
Autoconocimiento como competencia genérica en el Grado de Trabajo Social Self-knowledge as generic competence in social work education
}

\author{
Janet Romero Delgado ${ }^{1}$, Tomasa Báñez Tello² \\ janetromerodelgado@gmail.com, tomasabanez@ub.edu
}

\author{
${ }^{1}$ Estudiante de Trabajo Social, Unidad de \\ Formación e Investigación. Escuela de Trabajo \\ Social \\ Universidad de Barcelona \\ Barcelona, España
}

\begin{abstract}
Resumen La investigación cuyos resultados se presentan en esta comunicación, tiene como objetivo el análisis del trabajo de promoción del autoconocimiento, que de forma transversal se realiza en el Grado de Trabajo Social de la Universidad de Barcelona en el marco del Proyecto de innovación docente Tejiendo identidades profesionales. Autoconocimiento y Trabajo Social del Grupo Trans@net. Este análisis del trabajo de promoción del autoconocimiento puede contribuir a la mejora de esta iniciativa de innovación docente, así como a visibilizar la importancia de su incorporación como competencia profesional básica en la formación de Trabajo Social y de otros grados universitarios.
\end{abstract}

Palabras clave: Autoconocimiento, competencia genérica, Trabajo Social

Abstract The research whose results are presented in this paper, aims to analyse the work of promoting self-knowledge, which is carried out transversally in the Degree of Social Work of the University of Barcelona in the framework of the Teaching Innovation Project Weaving professional identities. Self-Knowledge and Social Work of Trans@net Group. This analysis of the work to promote selfknowledge can contribute to the improvement of this initiative of teaching innovation, as well as to make visible the importance of its incorporation as a basic professional competence in the social work education and in other university degrees.

\section{Keywords: Self-knowledge, generic competence, Social Work}

\section{INTRODUCCIÓN}

Los resultados de nuestro análisis del trabajo de promoción del autoconocimiento en el Grado de Trabajo Social de la Universidad de Barcelona son coherentes con la importancia que a nivel internacional ${ }^{1}$ se reconoce al autoconocimiento como exigencia para un ejercicio competente y responsable del trabajo social, así como con las experiencias de promoción en los estudiantes de trabajo social del autoconocimiento y la autoconciencia de su identidad de raza, etnia y género, orientación sexual, habilidades psíquicas y estatus

${ }^{1}$ En el documento Global standards for the education and training of the social workprofession, aprobado por la asamblea general de la Federación Internacional de Trabajadores Sociales y de la Federación Internacional de Escuelas de Trabajo Social en 2004 en Adelaida (Australia), http://ifsw.org/policies/global-standards/

\author{
${ }^{2}$ Profesora Titular de Universidad, Unidad de \\ Formación e Investigación. Escuela de Trabajo \\ Social \\ Universidad de Barcelona \\ Barcelona, España
}

socioeconómico, en el marco de una práctica profesional reflexiva (Cournoyer, 1996; Heydt, and Sherman, 2005; Knott-Scragg, 2007; Bender et al 2010; Gardner, 2001; Negi, et al, 2010). En este sentido, nuestros resultados muestran la valoración positiva que el alumnado participante en las actividades realiza en cuanto a la profundización de su autoconocimiento y autoconciencia, a nivel personal y en relación la realización de una práctica profesional reflexiva y responsable del trabajo social. No obstante, también se han puesto en evidencia los déficits en cuanto a la conexión y complementariedad del trabajo de las diferentes dimensiones del autoconocimiento, así como las dificultades organizativas y de contexto para promover el autoconocimiento de manera transversal en el itinerario formativo del alumnado.

\section{CONTEXTO}

La investigación cuyos resultados se presentan en esta comunicación, tiene como objetivo analizar el trabajo de autoconocimiento que de forma transversal se realiza en el Grado de Trabajo Social de la Universidad de Barcelona en el marco del Proyecto de innovación docente Tejiendo identidades profesionales. Autoconocimiento y Trabajo Social ${ }^{2}$ del Grupo Trans@net ${ }^{3}$. Este grupo formado por docentes del Grado de Trabajo Social tiene como objetivo innovar y mejorar la docencia, desde una perspectiva transdisciplinar y con la implicación del alumnado, a través de cuatro iniciativas innovadoras, entre las que se encuentra el proyecto de innovación docente Tejiendo identidades profesionales. Autoconocimiento y Trabajo Social. Este proyecto tiene como objetivo promover de manera transversal el desarrollo del autoconocimiento como competencia profesional básica en el alumnado del Grado de Trabajo Social de la Universidad de Barcelona y cuenta actualmente con la participación de 13 docentes de 12 asignaturas diferentes.

\footnotetext{
2 Proyecto de innovación docente reconocido y financiado por la Universidad de Barcelona http://mid.ub.edu/webpmid/content/teixintidentitats-professionals-autoconeixement-i-treball-social

${ }^{3}$ Grupo de Innovación Docente en transdisciplinariedad y aprendizaje en Trabajo Social. Grupo consolidado y reconocido por la Universidad de $\quad$ Barcelona http://mid.ub.edu/webpmid/content/transnet
} 
El autoconocimiento de los profesionales es fundamental desde la perspectiva reflexiva y relacional del trabajo social (Trevithick, 2003, 2011 y 2014; Howe, 1998; Murphy, et al. 2013). Un enfoque del trabajo social que implica el uso de uno mismo en el establecimiento de la relación profesional de ayuda y que exige a los profesionales el autoconocimiento y la autoconciencia respecto de sus valores personales y culturales, creencias, tradiciones y prejuicios, así como la capacidad de reflexión para identificar cómo estos valores y creencias pueden influir en su habilidad para establecer relaciones con las personas, y trabajar con diversos grupos de la población y de establecer estrategias de gestión de estas influencias en la realización de una práctica profesional competente.

La investigación cuyos resultados se presentan en está comunicación se justifica por la necesidad de evaluar los resultados de esta experiencia de innovación docente, a partir de las propias vivencias y opiniones del alumnado participante en la misma en relación con las mejoras de su autoconocimiento. Por otra parte, este análisis del trabajo de promoción del autoconocimiento realizado puede contribuir a la mejora de esta iniciativa de innovación docente, así como a visibilizar la importancia de su incorporación como competencia profesional genérica en la formación de grado de trabajo social y de otras disciplinas.

Dada la ausencia del autoconocimiento como competencia general en la formación universitaria de grado ${ }^{4} \mathrm{y}$ en la formación en trabajo social en España ${ }^{5}$, y el carácter pionero en España de nuestro proyecto, desde su inicio no solo hemos difundido sus resultados en diferentes congresos, sino que también hemos promovido la creación de un espacio de intercambio de experiencias sobre autoconocimiento y trabajo social, mediante la realización de una sesión paralela ${ }^{6}$ de trabajo del II Congreso Internacional de Trabajo Social realizado en Logroño en abril de 2016. Estos intercambios de experiencias nos han permitido comprobar la existencia de un creciente interés por trabajar el autoconocimiento tanto en la formación (Ariño et al, 2016; Canudas, 2016) como en el ejercicio profesional (Cazorla, 2016; Montagud, 2016), dada su relevancia para la realización de una práctica reflexiva del trabajo social.

\section{DESCRIPCIÓN}

Las actividades realizadas para el desarrollo del autoconocimiento, cuyos resultados se presentan en esta

\footnotetext{
${ }^{4}$ Esta competencia ausente en el Real Decreto 1393/2007, de 29 de octubre, por el que se establece la ordenación de las enseñanzas universitarias oficiales, se menciona en relación a la capacidad de los titulados universitarios de identificar sus propias necesidades formativas para el nivel de graduado en el Real Decreto 1027/2011, de 15 de julio, por el que se establece el Marco Español de Cualificaciones para la Educación Superior.

${ }^{5}$ En el documento aprobado en el 2007 por la Conferencia de Directores/as de Centros y Departamentos de Trabajo Social titulado La formación universitaria en Trabajo Social. Criterios para el diseño de planes de estudios de títulos de Grado en Trabajo Social, no aparece ninguna referencia explícita al autoconocimiento, aunque implícitamente está presente en competencias como la del desarrollo actitudes abiertas y empáticas basadas en el respeto y el reconocimiento ante la diversidad y la multiculturalidad.

${ }^{6}$ El diseño de la sesión paralela sobre El autoconocimiento: una estrategia de innovación docente en Trabajo Social coordinada por los profesores Josep María Mesquida y Belén Parra, está disponible en la página web del congreso https://cifets.unirioja.es/33/A6
}

comunicación, se han diseñado teniendo en cuenta la conceptualización del autoconocimiento y las estrategias para su desarrollo elaboradas por el profesorado promotor del proyecto. De acuerdo con esta conceptualización, el autoconocimiento es un proceso continuo y dinámico que, mediante el esfuerzo, la reflexión y la autoconciencia permite a la persona tener una percepción de sí misma, incluyendo aspectos intelectuales, emociones, valores éticos, capacidad de autonomía y deseos de autorrealización; a partir de la cual poder definir su identidad personal y desarrollar su personalidad. En nuestra conceptualización del autoconocimiento como competencia profesional del trabajo social, la autopercepción que la persona tiene de sí misma es la base para que se evalúe en relación con el trabajo social, identificando los aspectos que son congruentes con los valores y compromisos profesionales, sus potencialidades y/o aportes al trabajo social y las áreas en las que estos aspectos pueden influir negativamente en sus obligaciones profesionales y en la realización de una práctica profesional competente. Las estrategias para el desarrollo del autoconocimiento utilizadas en estas actividades incluyen la motivación e implicación de los estudiantes en el proceso de autoconocimiento, su acompañamiento mediante las tutorías y la supervisión individual y a través del diseño de un Proyecto de desarrollo personal y profesional que vertebra el proceso de aprendizaje , la autorreflexión sobre sí mismos, sobre sus experiencias personales y sobre las interacciones con personas con las que trabajaran como profesionales y la creación de un contexto docente facilitador (Báñez y Boixadós, 2017). El diseño y desarrollo detallado de estas actividades puede consultarse en las comunicaciones que para difundir los resultados del proyecto hemos presentado en diferentes congresos en diferentes congresos (Báñez, Matulic, Falcon y Boixadós, 2016; Báñez, Besa, García y Mas, 2016; García y Báñez, 2016; Báñez, y Boixadós, 2017; Boixadós, et al, 2017).

\section{Resultados}

La metodología de la investigación utilizada para evaluar el trabajo de autoconocimiento ha sido cualitativa y ha incluido el análisis de contenido de los diseños de las actividades de autoconocimiento elaborados por el profesorado (13 fichas), así como de las fichas de seguimiento en las que el alumnado participante (142 estudiantes) valora las mejoras en cuanto a su autoconocimiento. Por otra parte, se han realizado 12 entrevistas en profundidad a estudiantes participantes en las actividades de autoconocimiento con el objetivo de profundizar en cómo han experimentado el proceso de autoconocimiento y las potencialidades y debilidades que han identificado en el trabajo de promoción del autoconocimiento en el marco de su formación académica de grado.

El análisis de la información de las fichas de diseño de las actividades y de las fichas de seguimiento del alumnado, así como de las entrevistas, se ha realizado a partir de las categorías analíticas (Romero, 2017) basadas en la conceptualización del autoconocimiento y en la definición de sus dimensiones, con las que se trabaja en el proyecto de innovación docente (Báñez y Boixadós, 2017) y que se recogen en la Tabla 1 . 
Tabla 1. Resultados del desarrollo del autoconocimiento

\begin{tabular}{|c|c|c|}
\hline Dimensiones & Categorías & $\begin{array}{l}\text { \% } \\
\text { estudiantes } \\
\text { que han } \\
\text { mejorado } \\
\text { este aspecto }\end{array}$ \\
\hline \multirow[t]{6}{*}{ Reflexión personal } & Aspectos intelectuales. & 76,1 \\
\hline & Emociones & 50,9 \\
\hline & Actitud y valores éticos. & 27,8 \\
\hline & Capacidad de autonomía & 37 \\
\hline & $\begin{array}{l}\text { Deseos de autorrealización: } \\
\text { satisfacción con uno mismo y con el } \\
\text { entorno }\end{array}$ & 20 \\
\hline & Características y aptitudes personales & 37 \\
\hline \multirow{5}{*}{$\begin{array}{l}\text { Evaluación de la } \\
\text { identidad personal en } \\
\text { relación con el trabajo } \\
\text { social }\end{array}$} & $\begin{array}{l}\text { Asociación de valores personales con } \\
\text { la identidad profesional }\end{array}$ & 21,1 \\
\hline & $\begin{array}{l}\text { Potencialidades que dan lugar a una } \\
\text { buena práctica profesional }\end{array}$ & 35,1 \\
\hline & $\begin{array}{l}\text { Aportaciones que uno puede hacer al } \\
\text { Trabajo Social }\end{array}$ & 9,9 \\
\hline & $\begin{array}{l}\text { Dificultades que pueden influir } \\
\text { negativamente en la práctica } \\
\text { profesional }\end{array}$ & 20,53 \\
\hline & Intereses profesionales & 8,6 \\
\hline \multirow{9}{*}{$\begin{array}{l}\text { Estrategias de } \\
\text { desarrollo personal y } \\
\text { profesional }\end{array}$} & Proyección del yo futuro & 17,2 \\
\hline & $\begin{array}{l}\text { Mejora de la conexión con las propias } \\
\text { emociones }\end{array}$ & 6,6 \\
\hline & $\begin{array}{l}\text { Adquisición y/o ampliación de } \\
\text { conocimientos }\end{array}$ & 3,9 \\
\hline & Plausibilidad de los deseos personales & 1,3 \\
\hline & $\begin{array}{l}\text { Favorecer una mayor capacidad de } \\
\text { autonomía }\end{array}$ & 5,3 \\
\hline & Itinerario formativo y/o profesional & 9,2 \\
\hline & Favorecer prácticas competentes & 17,8 \\
\hline & $\begin{array}{l}\text { Desarrollar características y aptitudes } \\
\text { personales }\end{array}$ & 5,9 \\
\hline & Motivación personal/profesional & 18,5 \\
\hline
\end{tabular}

Presentamos a continuación los resultados más relevantes de la evaluación del trabajo de promoción del autoconocimiento a partir de la información recogida en la Tabla 1.

\section{A. El autoconocimiento en la formación de Trabajo Social en la Universidad de Barcelona}

Nuestros resultados muestran la necesidad de este trabajo de promoción del autoconocimiento, así como la adecuación de las estrategias utilizadas para su desarrollo. Los resultados en cuanto a las mejoras conseguidas muestran la satisfacción de alumnado por la contribución de las actividades de autoconocimiento a la configuración de su identidad personal y profesional, a la vez que ponen en evidencia la necesidad de profundizar en el desarrollo de las de desarrollo personal y profesional, una de las dimensiones del autoconocimiento.

\section{B. Impacto de la iniciativa innovadora}

El análisis del trabajo de promoción del autoconocimiento que hemos realizado a partir de los testimonios del alumnado participante en el proyecto de innovación docente muestra la diversidad de sus vivencias. De esta forma, hay estudiantes que manifiestan haber vivido la experiencia sin grandes dificultades, debido a que realizan este tipo de procesos de carácter introspectivo de forma cotidiana. Mientras otras personas han expresado que inicialmente tuvieron dificultades relacionadas con el clima de confianza necesario y con la ausencia de oportunidades en su formación académica para realizar este proceso de autoconocimiento. No obstante, una vez iniciado el proceso, todas las personas expresan sentirse satisfechas, enfatizando además el hecho de que se promueva el autoconocimiento de manera transversal y continuada dentro de la formación de Trabajo Social, debido a los beneficios aportados tanto a nivel personal, académico como profesional.
A nivel personal destacan la toma de conciencia acerca de uno/a mismo/a, el incremento de la autoestima y la seguridad personal, la identificación de cualidades personales y profesionales, la reflexión acerca de las influencias culturales e ideológicas a la hora de entender y posicionarse ante las diferentes situaciones, el reconocimiento de prejuicios e ideas preconcebidas y el conocimiento acerca de cómo uno se relaciona con los demás sujetos.

En cuanto a los beneficios académicos, señalan la toma de conciencia sobre los conocimientos adquiridos y las experiencias de aprendizaje vividas, así como la reflexión y diseño de su itinerario formativo y de su desarrollo profesional y el incremento de su capacidad crítica y reflexiva.

Finalmente, a nivel profesional, destacan como el trabajo de autoconocimiento ha contribuido a la configuración de su identidad profesional y a la definición de su rol y práctica profesional, en coherencia con sus valores personales y del Trabajo Social, incrementando su seguridad personal y permitiéndoles valorar su idoneidad para ser profesionales del Trabajo Social. Por otra parte, este trabajo les ha ayudado en la comprensión de su propia subjetividad y en la toma de conciencia acerca de las influencias de sus características personales, emociones y valores en el momento de establecer la relación de ayuda profesional; así como a establecer los mecanismos para que su subjetividad no influya negativamente en la realización de una práctica profesional competente y responsable.

\section{B. Elementos clave en la promoción del autoconocimiento como competencia genérica}

Además de evaluar los beneficios que para la mejora de su autoconocimiento ha tenido esta iniciativa de innovación docente, el alumnado participante en el mismo nos ha expresado, a través de las fichas de seguimiento y de las entrevistas cuales son los elementos clave, en relación al alumnado, al profesorado y al contexto, para el desarrollo del autoconocimiento como competencia genérica.

Los elementos clave en relación con el contexto, identificados a partir de los testimonios del alumnado, para trabajar en la promoción del autoconocimiento como competencia genérica en la formación académica superior son los siguientes.

\section{Compartir visiones y experiencias entre compañeros}

Expresan que el hecho de compartir experiencias y pensamientos entre compañeros/as favorece la reflexión e introspección acerca de la identidad tanto personal como profesional. No obstante, creen que para ello es fundamental que se establezca un clima de confianza en el aula para favorecer este intercambio de pensamientos, experiencias, etcétera. En este sentido, las propuestas que realizan para la mejora de estas cuestiones incluyen el favorecer la creación de más espacios de debate y de discusión, la incorporación de la visión que los/as otros/as tienen de ellos/as, contrastando de esta forma la autoimagen con la visión de que de ellos/as tiene los demás. En cuento al clima de confianza en el aula consideran fundamental que las clases tengan un número reducido de estudiantes (10-15 personas) y que se trabaje en asignaturas anuales, lo que facilitaría la creación de relaciones más estrechas entre los/as participantes. 


\section{Trabajar las evidencias fuera del espacio aula}

Los estudiantes consideran que para realizar un proceso introspectivo como el que requiere el autoconocimiento, es preciso poder disponer de un espacio tranquilo y confortable, afirmando que no todos los momentos son válidos y que consideran que las evidencias que han de entregar sobre su proceso de autoconocimiento se elaboren previamente a la realización de la clase en el aula.

\section{Sesiones a nivel individualizado}

A pesar de que los estudiantes enfatizan los beneficios que genera el compartir y trabajar en grupo, también señalan la necesidad de que simultáneamente se establezcan sesiones individualizadas que permitan profundizar en los diferentes aspectos del autoconocimiento a un nivel más íntimo. Una de las propuestas para facilitar la viabilidad de estos momentos de acompañamiento individualizado al alumnado por parte del profesorado, sería utilizar el Plan de Acción Tutorial del grado, adoptando sus contenidos y organización a este trabajo de promoción del autoconocimiento.

Los elementos clave en relación con el profesorado, identificados a partir de los testimonios del alumnado, para trabajar en la promoción del autoconocimiento como competencia genérica en la formación académica superior son los siguientes.

\section{Experiencia y capacidad de guía en el proceso de autoconocimiento}

El autoconocimiento es un proceso complejo e inacabado, razón por la que los estudiantes señalan la relevancia de que el profesorado haya trabajado su propio proceso autoconocimiento, con el fin de identificar y establecer guías que ayuden y orienten al alumnado a partir de un saber vivencial. También consideran fundamental que el profesorado explique la importancia del proceso del autoconocimiento y motive al alumnado, favoreciendo de esta forma su implicación.

\section{Relación horizontal, empática y no autoritaria con los/as estudiantes}

En coherencia con la importancia de la existencia de un clima y vínculo de confianza para favorecer este tipo de procesos, el alumnado considera que el profesorado se debe mostrar una actitud empática y no juzgadora, con el propósito de que los/as alumnos/as puedan expresarse con sinceridad y sin miedo al rechazo. Así mismo, el alumnado considera positivo que se establezca una relación de horizontalidad que ayude a generar un vínculo de proximidad.

Los elementos clave en relación con los propios estudiantes, identificados a partir de sus testimonios, para trabajar en la promoción del autoconocimiento como competencia genérica en la formación académica superior son los siguientes.

\section{El dominio del lenguaje emocional}

Varias personas han puesto en manifiesto la falta de dominio sobre el propio lenguaje para expresar y nombrar lo que sienten, con lo que se complica el trabajo introspectivo a realizar. Por ello, plantean que podría ser beneficioso trabajar e incrementar los conocimientos acerca de la inteligencia emocional y efectuar talleres en relación a las emociones.
La motivación por parte del alumnado y que se trate de un proceso voluntario

Para desarrollar un proceso introspectivo es fundamental que el alumnado se implique personalmente y tenga motivación para hacerlo, ya que sino no tendrá sentido ejecutar este tipo de proceso. Por ese motivo, los/as estudiantes consideran que este tipo de actividades deberían de ser voluntarias, ya que hacerlas sin motivación y sin profundizar carece de sentido. También plantean que la honestidad personal es necesaria para poder identificar sus valores y características personales, sus potencialidades y limitaciones para una práctica competente del trabajo social, pero podría verse comprometida por el miedo de los estudiantes a ser juzgados como parte del proceso de evaluación de sus aprendizajes, ya que las actividades se realizan en el marco de su proceso de formación académica formal como trabajadores/as sociales.

\section{CONCLUSIONES}

El análisis y evaluación del trabajo de promoción del autoconocimiento como competencia genérica que se realiza en el grado de Trabajo Social en la Universidad en Barcelona, nos ha permitido identificar los elementos clave y las estrategias que explican la valoración positiva y los beneficios señalados por el alumnado participante en cuanto a la profundización de su autoconocimiento y autoconciencia, a nivel personal y en relación la realización de una práctica profesional reflexiva y responsable del trabajo social. No obstante, también se han señalado propuestas de mejora que pretenden mejorar la conexión y complementariedad del trabajo de las diferentes dimensiones del autoconocimiento, así como las dificultades organizativas y de contexto para promover el autoconocimiento de manera transversal en el itinerario formativo del alumnado.

Dado que el proyecto de innovación docente tiene prevista su finalización en el 2019, el profesorado participante en el mismo incorporará estas propuestas de mejora al trabajo de promoción del autoconocimiento, a la vez que planteará a los responsables académicos de la titulación su inclusión como competencia genérica del grado de Trabajo Social en la Universidad en Barcelona y la elaboración de un mapa de competencias transversales que permita su desarrollo en la formación académica. Finalmente, la presentación y difusión de los resultados de esta iniciativa innovadora puede contribuir a visibilizar la importancia del autoconocimiento, así como motivar y orientar a otros docentes para su incorporación como competencia genérica en la formación académica superior. De esta forma, se facilitará que los/as futuros/as profesionales además de adquirir una formación sólida y rigurosa a nivel teórico y metodológico, tengan oportunidades para desarrollar competencias genéricas y transversales, que como el autoconocimiento puedan contribuir a la construcción de su identidad personal y profesional, desde una perspectiva crítica y reflexiva.

\section{REFERENCIAS}

Ariño, E., Ovejas, R. y Berasaluze, A. (2016). Construyendo profesionales a través del autoconocimiento dialogado, en Carbonero, D. Raya, E. Caparros, N. y Gimeno, C. (Coords), Respuestas transdisciplinares en una sociedad global. Aportaciones desde el Trabajo Social, Libro de Actas del II Congreso Internacional de Trabajo Social, 
Universidad de La Rioja, Logroño. Recuperado de https://publicaciones.unirioja.es/catalogo/online/CIFETS_2 016/Monografia/pdf/TC419.pdf

Báñez, T., Besa, S., García, E. y Mas, A. (2016). Autoconocimiento y trabajo social reflexivo. De cómo coprotagonizar una práctica dialogada del trabajo social. En Guinot, C., Ferran, A. (eds), Trabajo social: arte para generar vínculos, Libro de Actas del III Congreso Internacional: Trabajo Social, arte para generar vínculos. Universidad de Deusto, San Sebastián. Recuperado de http://www.deusto-

publicaciones.es/deusto/index.php/es/otraspubes/otraspub01c

Báñez, T., Matulic M ${ }^{\mathrm{a}}$ V., Falcon, A. M. y Boixadós, A. (2016). Tejiendo identidades. Autoconocimiento y trabajo social, en Carbonero, D. Raya, E. Caparros, N. y Gimeno, C. (Coords), Respuestas transdisciplinares en una sociedad global. Aportaciones desde el Trabajo Social, Libro de Actas del II Congreso Internacional de Trabajo Social, Universidad de La Rioja, Logroño. Recuperado de https://publicaciones.unirioja.es/catalogo/online/CIFETS_2 016/Monografia/pdf/TC417.pdf

Báñez, T. y Boixadós, A. (2017). Conceptualización del autoconocimiento como competencia profesional básica del trabajo social, en Bolívar, A. (Presidencia), VI Congreso Internacional Multidisciplinar de Investigación Educativa, Asociación Multidisciplinar de Investigación Educativa (AMIE), 29 y 30 de junio, Bilbao (España)

Bender, K., Negi, N. and Fowler, D. N. (2010). Exploring the relationship between self-awareness and student commitment and understanding of culturally responsive social work practice. Journal of ethnic \& cultural diversity in social work, 19(1), 34-53.

Boixadós, A; Munté, A.; De Vicente, I.; Matulič, MV; Báñez, T. (2017). El autoconocimiento en el espacio de la supervisión educativa, en los estudios de trabajo social de la Universidad de Barcelona, en González, M.; Raposo, M.; Erkizia, A.; Cebrián, M.; Pérez, A.; Barberá, M.A.; Canet, O.; Zabalza, M.A. (Coords), Recursos para un Prácticum de Calidad Libro de Actas del XIV Symposium Internacional sobre el practicum y las prácticas externas, Asociación para el Desarrollo del Prácticum y de las Prácticas Externas, Poio (Pontevedra: Red de Prácticum (REPPE).

Canudas, A. (2016). Herramientas pedagógicas para facilitar el autoconocimiento del alumnado, en Carbonero, D. Raya, E. Caparros, N. y Gimeno, C. (Coords), Respuestas transdisciplinares en una sociedad global. Aportaciones desde el Trabajo Social, Libro de Actas del II Congreso Internacional de Trabajo Social, Universidad de La Rioja, Logroño.

Recuperado https://publicaciones.unirioja.es/catalogo/online/CIFETS 2 016/Monografia/pdf/TC425.pdf

Cazorla, J. (2016). El conocimiento de uno mismo como herramienta de ayuda: Análisis de una experiencia práctica dentro del Grado de Trabajo Social, en Carbonero, D. Raya, E. Caparros, N. y Gimeno, C. (Coords), Respuestas transdisciplinares en una sociedad global. Aportaciones desde el Trabajo Social, Libro de Actas del II Congreso
Internacional de Trabajo Social, Universidad de La Rioja, Logroño. Recuperado de https://publicaciones.unirioja.es/catalogo/online/CIFETS_2 016/Monografia/pdf/TC414.pdf

Cournoyer, B. (1996). The Social work skills workbook. Belmont (USA): Pacific Grove: Brooks/Cole.

García, E. y Báñez, T. (2016). Tejiendo identidades profesionales. Un proceso creativo y transformador, en Londoño Monroy, G.; Rodríguez Illera , J.L. (comps.) (2017). Relatos Digitales en Educación Formal y Social. Barcelona: Universitat de Barcelona. DOI: 10.1344/105.000003160. Recuperado de http://www.greav.net/descargas/Actas2016.pdf

Gardner, F. (2001). Social Work Students and Self-awareness: how does it happen? Reflective Practice, 2(1), 27-40.

Heydt, M. J. and Sherman, N. E. (2005). Conscious Use of Self: Tuning the Instrument of Social Work Practice with Cultural Competence, The Journal of Baccalaureate Social Work, Vol. 10 (2), 25-40

Howe, D. (1998). Relationship-based thinking and practice in social work. Journal of Social Work Practice, 12(1), 4556.

Knott, C. and Scragg, T. (2007). Reflective practice in social work, Exeter: Learning, Matters.

Montagud, X. (2016). Reflexionar para conocer: la autoetnografía, un instrumento para el (auto)conocimiento de la relación profesional, III Congreso Internacional de trabajo social trabajo social: arte para generar vínculos, Universidad de Deusto, San Sebastián. Recuperado de http://www.deustopublicaciones.es/deusto/index.php/es/otraspubes/otraspub01c

Murphy, D., Duggan, M. and Joseph, S. (2013). Relationshipbased social work and its compatibility with the personcentred approach: Principled versus instrumental perspectives. British Journal of Social Work, 43(4), 703719.

Negi, N. J., Bender, K. A., Furman, R., Fowler, D. N. and Prickett, J. C. (2010). Enhancing self-awareness: A practical strategy to train culturally responsive social work students. Advances in Social Work, 11(2), 223-234.

Romero, J. (2017). Autoconocimiento y Trabajo Social. Análisis del autoconocimiento en la formación de Grado en Trabajo Social en la Universidad de Barcelona. Trabajo de fin de grado. Universidad de Barcelona, Barcelona (España).

Trevithick, P. (2003). Effective relationship-based practice: a theoretical exploration. Journal of Social Work Practice, 17(2), 163-176.

Trevithick, P. (2011). Social work skills and knowledge: A practice handbook. Maidenhead: McGraw-Hill Education (UK).

Trevithick, P. (2014). Humanising managerialism: reclaiming emotional reasoning, intuition, the relationship, and knowledge and skills in social work. Journal of Social Work 28(3), 287-311. 